\title{
Over All Assessment of Tribals of Udaipur under Livestock Development Service of Tribal Area Development Programme
}

\author{
Sonali Sharma ${ }^{*}$, Snehlata Maheshwari², Rajshree Upadhyay², \\ Bhupendra Upadhyay ${ }^{3}$ and B.L. Dangi ${ }^{4}$ \\ ${ }^{1}$ SMS (Home Science), KVK-Barmer (Raj.), India \\ ${ }^{2}$ Department of Home Science Extension and Communication Management, College of Home \\ Science MPUAT, Udaipur, Rajasthan, India \\ ${ }^{3}$ Department of Statistics, Rajasthan College of Agriculture, MPUAT, Udaipur, Raj., India \\ ${ }^{4} S M S$ (Animal Science), KVK-Barmer (Raj.), India \\ *Corresponding author
}

\section{A B S T R A C T}

\section{Keywords}

Knowledge, Adoption, Opinion, Constraints

Article Info

Accepted:

04 August 2018

Available Online:

10 September 2018
The present study was undertaken with the objectives to find out the knowledge and adoption of improved practices promoted under Livestock Development service of TADP among the beneficiaries, opinion of beneficiaries towards the service promoted under TADP and to explore the constraints faced by the beneficiaries of the TADP. The study was conducted in purposively selected Jadol block of Udaipur district. The sample consisted of 60 respondents (30 beneficiaries and 30 non-beneficiaries). Result of the study reveals that knowledge and adoption of beneficiaries as compared to non-beneficiaries were high and significant difference was found. Beneficiaries were in favourable opinion and were having constraints to great extent.

\section{Introduction}

The tribals forms an integral part of India's social fabric and has second largest concentration of tribal population in the world after that of the African continent.

In general tribal societies are small in scale, are restricted in the spatial and temporal range of their social, legal and political relations and possess a morality, a religion and world view of corresponding dimensions. The Indian constitution enjoin on the state the responsibility to promote, with special care, the education and economic interests of scheduled tribes and protect them from social injustice and all forms of exploitation. Thus their development is a special responsibility of the state. With the view to bring the tribal areas or people of the country in the mainstream of economic development and accelerating the pace of socio-economic development, the State Government has made significant efforts to uplift the tribal people and started various activities for their social and economic development. A special programme, known as Tribal Area Developmental Programme (TADP) was taken 
up, on a pilot basis under a central sector plan scheme of the Ministry of Agriculture in 1964.

The tribes of Rajasthan, as elsewhere in the country are passing through a process of socio-cultural and demographic transformation. Progress in livestock sector is directly related to a more balanced development of rural economy and upliftment of poorer sections of the society. Straws, stovers and other agricultural by products would continue to be major inputs as livestock feed for ruminants. There is a large shortage of green fodder (45 to 50 per cent) and concentrates for livestock feeding. The programmes on production of feed and fodder are being further strengthened both for increasing bio- mass availability and enrichment of straws and stovers.

These activities play a vital role in providing household nutritional security, health security by supplying nutrition through balanced diet and increased employment and income. Animals also provide a diverse range of output for cultivation, irrigation, transport; fibre and leather goods, manure for fertilizer and fuel besides direct production of milk, meat and eggs for human consumption. Thus these Agriculture services provide economic security and social status to the family. Our country has achieved self-sufficiency and a good degree of stability of food production but agricultural practices are still performed by using primitive tools and methods, which have been in use for centuries. Though agriculture is the backbone of India's economy yet technological innovation to change the primitive method of cultivation is at infancy among the farm women folk of country and this may be due to their limited knowledge and skill in application of science and technology to daily living, resulting in fatigue and drudgery at every moment. In order to improve the efficiency and productivity as well as reduce drudgery of the farm women, there is a need to make them aware of small, simple, low cost, hand operated tools.

Development programmes are many but to what extent they have benefitted the tribal's? Are these programmes really worth for them? It is important to study about tribal's, where do the tribal's stand today? Are they benefitted by the government measures? Does a tribal adopt the services for their betterment? What is their opinion about the programmes? What problems they are facing? Hence, it was considered worthwhile to explore the idea to find out the "Impact of Agricultural Services of Tribal Area Development Programme on Tribals of Udaipur district".

\section{Materials and Methods}

The study was conducted in Udaipur district of Rajasthan as for the development of tribals a separate department i.e. Tribal Area Development Department is in function. The department implements various schemes or programmes for tribal development along with Agricultural services (Livestock Development). The service was implemented by the NGO-BAIF (Bharatiya Agro Industries Foundation) known as RRIDMA (Rajasthan Rural Institute of Development Management) in Jadol block to facilitate the tribal beneficiaries for Tribal Area Development Department. For the service, two villages (odha, magwas) were selected randomly for the beneficiaries from programme implementing villages and similarly two villages (kherwada, bhuja) were selected for non-beneficiaries from non programme villages. Thus in total 4 villages were selected for the study purpose. Thirty beneficiaries and non-beneficiaries were selected randomly, the total sample for the study comprised of 60 respondents. For the analysis of data, frequency, percentage, mean percent score, mean weighted score, student ' $t$ ' test and paired ' $t$ ' test were used. 


\section{Results and Discussion}

\section{Background Information}

Age

Data presented in Table 1 reveal that 46.67 per cent of the beneficiaries and 38.33 per cent of the non-beneficiaries belonged to 46-60 years of age group followed by 40.00 per cent of the beneficiaries and 32.78 per cent of the nonbeneficiaries belonged to 31-45 years of age group.

\section{Occupation of the respondents}

All the beneficiaries and non-beneficiaries of the services were involved in farming and livestock occupation.

\section{Education}

Perusal of Table 1 reveals that 50.00 per cent of the beneficiaries and 43.33 per cent of the non beneficiaries were educated up to primary level, whereas, 30.00 per cent of the beneficiaries and 33.33 per cent of the nonbeneficiaries were illiterate.

\section{Caste}

All the beneficiaries and non-beneficiaries belonged to scheduled tribe only as the TADP is meant for tribals only.

\section{Family type}

It is evident from Table 2 that 60.00 per cent of the beneficiaries and non-beneficiaries were from the nuclear family while 40 per cent of the beneficiaries and non-beneficiaries were from joint family.

\section{Family size}

The table further shows that 33.33 per cent of the beneficiaries and 30 per cent of the non- beneficiaries had medium size family (5 to 8 members) followed by 40 per cent beneficiaries and 30 per cent non beneficiaries belonged to small family (upto 4 members) and only 13.33 per cent beneficiaries and 33.33 per cent non-beneficiaries were having large family ( 9 and more).

\section{Family occupation}

Table 2 depicts that agriculture and livestock were the main family occupation of all the beneficiaries and non-beneficiaries (100\%).

\section{Land holding}

Table 3 reveals that marginal land i.e. 2.5 big has land possessed by 40 per cent beneficiaries and 63.33 per cent of the nonbeneficiaries followed by 36.66 per cent of the beneficiaries who having $5.0-9.5$ big has and 30.00 per cent of the non - beneficiaries having $2.5-5.0 \mathrm{big}$ has of land.

\section{Housing}

With reference to housing 53.33 per cent of beneficiaries and 66.66 per cent non beneficiaries were residing in Kachha house followed by 30.00 per cent of the beneficiaries and 23.33 per cent of the non-beneficiaries residing in mixed houses. Only 16.66 per cent beneficiaries and 10.00 per cent non beneficiaries were residing in pucca houses.

\section{Livestock ownership}

Table 3 reveals that 13.33 per cent beneficiaries and 30.00 per cent nonbeneficiaries had medium herd size consisting of 4 to 6 milch animals and one third of beneficiaries $(53.33 \%)$ and non-beneficiaries $(36.66 \%)$ had large herd size.

There were 33.33 per cent beneficiaries and 30.00 per cent non-beneficiaries had small herd size animals. 


\section{Dwelling for livestock}

Regarding dwelling for livestock, 70 per cent beneficiaries and non-beneficiaries had open dwelling for livestock while 16.6 per cent beneficiaries and one-third non-beneficiaries were having thatched or katcha dwelling for livestock. Only 13.33 per cent of the beneficiaries had pucca dwelling for livestock.

\section{Socio-economic status}

On the basis of scores obtained by the respondents in different aspects of SES scale, the respondents were categorized in high, medium and low socio-economic status. Table 4 depicts that all the beneficiaries and nonbeneficiaries belonged to low socio economic status.

\section{Knowledge of the respondents about general information regarding livestock development service}

This part takes care of information about the service like starting year of the service, its beneficiaries, main objectives and activities of the service. The knowledge of beneficiaries and non - beneficiaries about information related to livestock service of TADP is presented in Table 5. Perusal of the table reveals that, beneficiaries possessed good knowledge for the development as depicted by MPS 82.85 and non-beneficiaries had MPS 52.32 depicting average knowledge, distribution of the respondents by knowledge category reveals that 86.66 per cent of the beneficiaries possessed good knowledge and 63.33 per cent of the non-beneficiaries possessed average knowledge regarding general information of the service.

Perusal of the table illustrate that highly significant difference was found in the knowledge of beneficiaries and nonbeneficiaries in the services. Thus, it can be inferred that the knowledge of the beneficiaries was more as compared to nonbeneficiaries about starting year of the service, criteria for selecting beneficiaries, objectives and activities of each service.

This is obvious as the beneficiaries were part of the programme, availing benefits, definitely has to have higher knowledge than the nonbeneficiaries.

\section{Knowledge of improved practices promoted under livestock development services by the respondents}

An effort was also made to see the knowledge of improved practices promoted under the service by the respondents. In the service, the respondent's knowledge was assessed on number of improved practices and was grouped under various components of the service.

For Livestock Development, beneficiaries were given facilities of free artificial insemination, free seeds for green fodder and provided trainings on regular basis with respect to animals feeds, health care practices etc.

With regard to overall knowledge of Livestock Development (Table 6) beneficiaries had good knowledge with MPS 84.79. Distribution of respondents also reveals that 80 per cent beneficiaries were in good knowledge category and 20.00 per cent beneficiaries were in average knowledge category.

In case of non-beneficiaries, MPS 59.21 depicting average knowledge and less than half of the non-beneficiaries (43.33\%) were in good knowledge category, while 33.33 per cent and 23.33 per cent of the nonbeneficiaries were in average and poor knowledge category respectively leading to average knowledge scores. 
Data presented in the table depicts the knowledge of respondents regarding components of livestock development and in all the four components of livestock development viz. breeding, feeding, milk production and health care, beneficiaries had good knowledge with MPS ranging 78.66 84.90 while non- beneficiaries had average knowledge with MPS ranging between 50.00 and 64.11 i.e. higher side of the average knowledge. This is also evident from the distribution of the respondents in extent of knowledge regarding livestock development that majority of the beneficiaries were falling in good knowledge category (73.33 - 83.33\%) and very few beneficiaries were in average $(16.66-23.33 \%)$ and poor knowledge category $(6.66-13.33 \%)$.

However, in livestock development, majority of the non-beneficiaries were in good knowledge category (36.66 - 63.33\%) but $10.00-36.66$ per cent of the non-beneficiaries were in average and $6.66-33.33$ per cent of the non-beneficiaries were in poor knowledge category, leading to average knowledge of non-beneficiaries in all the components.

The reasons behind the wide range of difference in knowledge of the beneficiaries and the non-beneficiaries may be that beneficiaries were more aware about various aspects viz. improved breeds, artificial insemination methods with advantages, conceiving and heating time, calving practices, their proper feed at various stages, ratio of feed mixture, importance of green fodder, method to increase nutritive value of fodder, colostrums advantages, vaccination against diseases, deworming, milking method with sanitary precaution as compared to nonbeneficiaries.

Table.1 Distribution of the respondents according to their personal characteristics $n=60$

\begin{tabular}{|c|c|c|c|}
\hline \multirow{2}{*}{$\begin{array}{c}\text { S. } \\
\text { No. }\end{array}$} & \multirow[t]{2}{*}{ Variables } & Beneficiaries $n_{1}=180$ f ( $\left.\%\right)$ & Non-Beneficiaries $n_{2}=180$ f ( \% ) \\
\hline & & LDC $n=30$ & LDC $n=30$ \\
\hline 1 & \multicolumn{3}{|l|}{ Age } \\
\hline $\mathbf{A}$ & $18-30$ years & $\begin{array}{c}5 \\
(16.66)\end{array}$ & $\begin{array}{c}10 \\
(33.33)\end{array}$ \\
\hline $\mathbf{B}$ & $31-45$ years & $\begin{array}{c}11 \\
(36.66)\end{array}$ & $\begin{array}{c}8 \\
(26.66)\end{array}$ \\
\hline $\mathbf{C}$ & $46-60$ years & $\begin{array}{c}14 \\
(46.66)\end{array}$ & $\begin{array}{c}12 \\
(40.00)\end{array}$ \\
\hline 2 & \multicolumn{3}{|l|}{ Occupation } \\
\hline$\overline{\mathbf{A}}$ & $\begin{array}{l}\text { Farming } \quad \& \\
\text { Livestock }\end{array}$ & $\begin{array}{c}30 \\
(100)\end{array}$ & $\begin{array}{c}30 \\
(100)\end{array}$ \\
\hline 4 & \multicolumn{3}{|l|}{ Education } \\
\hline$\overline{\mathbf{A}}$ & Illiterate & $\begin{array}{c}9 \\
(30.00)\end{array}$ & $\begin{array}{c}10 \\
(33.33)\end{array}$ \\
\hline $\mathbf{B}$ & Can sign & $\begin{array}{c}3 \\
(10.00)\end{array}$ & $\begin{array}{c}6 \\
(20.00)\end{array}$ \\
\hline $\mathbf{C}$ & Read and write & $\begin{array}{c}2 \\
(6.66)\end{array}$ & $\begin{array}{c}2 \\
(6.66)\end{array}$ \\
\hline D & Primary & $\begin{array}{c}15 \\
(50.00)\end{array}$ & $\begin{array}{c}13 \\
(43.33)\end{array}$ \\
\hline
\end{tabular}


Table. 2 Distribution of the respondents by their social characteristics $n=60$

\begin{tabular}{|c|c|c|c|}
\hline \multirow{2}{*}{$\begin{array}{c}\text { S. } \\
\text { No. }\end{array}$} & \multirow[t]{2}{*}{ Variables } & Beneficiaries $n_{1}=180$ f ( \% ) & Non-Beneficiaries $n_{2}=180$ f ( \%) \\
\hline & & LDC $n=30$ & LDC $n=30$ \\
\hline 1 & Family Type & & \\
\hline $\mathbf{A}$ & Nuclear & $\begin{array}{c}18 \\
(60.00)\end{array}$ & $\begin{array}{c}18 \\
(60.00)\end{array}$ \\
\hline $\mathbf{B}$ & Joint & $\begin{array}{c}12 \\
(40.00)\end{array}$ & $\begin{array}{c}12 \\
(40.00)\end{array}$ \\
\hline 2 & Family Size & & \\
\hline $\mathbf{A}$ & $\begin{array}{l}\text { Small (upto } 4 \\
\text { Members) }\end{array}$ & $\begin{array}{c}12 \\
(40.00)\end{array}$ & $\begin{array}{c}9 \\
(30.00)\end{array}$ \\
\hline B & Medium (5- 8 Members) & $\begin{array}{c}16 \\
(53.33)\end{array}$ & $\begin{array}{c}11 \\
(36.66)\end{array}$ \\
\hline C & $\begin{array}{l}\text { Large } \\
\text { (9 and more) }\end{array}$ & $\begin{array}{c}4 \\
(13.33)\end{array}$ & $\begin{array}{c}10 \\
(33.33)\end{array}$ \\
\hline 3 & Family Occupation & & \\
\hline$\overline{\mathbf{A}}$ & $\begin{array}{l}\text { Agriculture } \\
\text { Alone }\end{array}$ & $\begin{array}{c}30 \\
(100)\end{array}$ & $\begin{array}{c}30 \\
(100)\end{array}$ \\
\hline $\mathbf{B}$ & $\begin{array}{l}\text { Agriculture+ } \\
\text { Artisan/ } \\
\text { Craftsman }\end{array}$ & $\begin{array}{c}6 \\
(20.00)\end{array}$ & $\begin{array}{c}11 \\
(36.66)\end{array}$ \\
\hline
\end{tabular}

Table.3 Distribution of the respondents by their economic characteristics $n=60$

\begin{tabular}{|c|c|c|c|}
\hline S. No. & Variables & Beneficiaries $\mathrm{f}(\%) \mathrm{n}=\mathbf{3 0}$ & Non-Beneficiaries $\mathbf{f}(\%) \mathrm{n}=30$ \\
\hline 1 & Land Holding (in bighas) & & \\
\hline $\mathbf{A}$ & Marginal (upto 2.5) & $\begin{array}{c}12 \\
(40.00)\end{array}$ & $\begin{array}{c}19 \\
(63.33)\end{array}$ \\
\hline$\overline{\mathbf{B}}$ & Small $(2.5-5.0)$ & $\begin{array}{c}7 \\
(23.33)\end{array}$ & $\begin{array}{c}9 \\
(30.00)\end{array}$ \\
\hline $\mathbf{C}$ & Medium $(5.0-9.5)$ & $\begin{array}{c}11 \\
(36.66)\end{array}$ & $\begin{array}{c}2 \\
(6.66)\end{array}$ \\
\hline 2 & Housing & & \\
\hline $\mathbf{A}$ & Kachha House & $\begin{array}{c}16 \\
(53.33)\end{array}$ & $\begin{array}{c}20 \\
(66.66)\end{array}$ \\
\hline $\mathbf{B}$ & Mixed house & $\begin{array}{c}9 \\
(30.00)\end{array}$ & $\begin{array}{c}7 \\
(23.33)\end{array}$ \\
\hline $\bar{C}$ & Pukka House & $\begin{array}{c}5 \\
(16.66)\end{array}$ & $\begin{array}{c}3 \\
(10.00)\end{array}$ \\
\hline 3 & Live stock Ownership & & \\
\hline$\overline{\mathbf{A}}$ & $\begin{array}{l}\text { Small herd size }(1-3 \\
\text { milch animals) }\end{array}$ & $\begin{array}{c}10 \\
(33.33)\end{array}$ & $\begin{array}{c}9 \\
(30.00)\end{array}$ \\
\hline $\bar{B}$ & $\begin{array}{l}\text { Medium herd size (4-6 } \\
\text { milch animals) }\end{array}$ & $\begin{array}{c}4 \\
(13.33)\end{array}$ & $\begin{array}{c}10 \\
(30.00)\end{array}$ \\
\hline $\mathbf{C}$ & $\begin{array}{l}\text { Large herd size (More } \\
\text { than } 6 \text { milch animals) }\end{array}$ & $\begin{array}{c}16 \\
(53.33)\end{array}$ & $\begin{array}{c}11 \\
(36.66)\end{array}$ \\
\hline 4 & Dwelling for Livestock & & \\
\hline$\overline{\mathbf{A}}$ & Open/nil & $\begin{array}{c}11 \\
(36.66)\end{array}$ & $\begin{array}{c}21 \\
(70.00)\end{array}$ \\
\hline $\mathbf{B}$ & Thatched/kutcha & $\begin{array}{c}17 \\
(56.66)\end{array}$ & $\begin{array}{c}9 \\
(30.00)\end{array}$ \\
\hline $\mathrm{C}$ & Pucca & $\begin{array}{c}2 \\
(6.66)\end{array}$ & 0 \\
\hline
\end{tabular}


Table.4 The beneficiaries and non-beneficiaries belonged to low socio - economic status

\begin{tabular}{|c|c|c|c|}
\hline S. No. & Variables & Beneficiaries $\mathbf{f}(\%) \mathrm{n}=\mathbf{3 0}$ & Non-Beneficiaries $\mathbf{f}(\%) \mathrm{n}=\mathbf{3 0}$ \\
\hline \multicolumn{4}{|c|}{ Socio - Economic Status } \\
\hline 1 & High & 0 & 0 \\
\hline 2 & Medium & 0 & 0 \\
\hline 3 & Low & $30(100)$ & $30(100)$ \\
\hline
\end{tabular}

Table.5 Distribution of the respondents by the Knowledge about Agricultural services $n=60$

\begin{tabular}{|c|c|c|c|c|c|c|c|c|c|c|}
\hline \multirow{3}{*}{$\begin{array}{l}\text { S. } \\
\text { No. }\end{array}$} & \multirow[t]{3}{*}{ Services } & \multicolumn{8}{|c|}{ Knowledge } & \multirow{3}{*}{ t value } \\
\hline & & \multicolumn{3}{|c|}{ Beneficiaries $\mathrm{n}_{1}=30 *$} & $f(\%)$ & \multicolumn{4}{|c|}{ Non-Beneficiaries $\mathbf{n}_{2}=30 * \mathbf{f}(\%)$} & \\
\hline & & Good & Average & Poor & MPS & Good & Average & Poor & MPS & \\
\hline 1 & $\begin{array}{l}\text { Livestock } \\
\text { Development }\end{array}$ & $\begin{array}{c}26 \\
(86.66)\end{array}$ & $\begin{array}{c}4 \\
(13.33)\end{array}$ & 0 & 82.85 & $\begin{array}{c}7 \\
(23.33)\end{array}$ & $\begin{array}{c}19 \\
(63.33)\end{array}$ & $\begin{array}{c}4 \\
(13.33)\end{array}$ & 52.38 & $9.02 * *$ \\
\hline
\end{tabular}

Table.6 Distribution of the respondents by the knowledge regarding improved practices of Livestock Development $n=60$

\begin{tabular}{|c|c|c|c|c|c|c|c|c|c|}
\hline \multirow[t]{3}{*}{ S. No } & \multirow[t]{3}{*}{ Components } & \multicolumn{8}{|c|}{ Knowledge } \\
\hline & & \multicolumn{4}{|c|}{ Beneficiaries $n_{1}=30 \mathrm{f}(\%)$} & \multicolumn{4}{|c|}{ Non - Beneficiaries $n_{2}=30 \mathrm{f}(\%)$} \\
\hline & & Good & Average & Poor & MPS & Good & Average & Poor & MPS \\
\hline 1 & Breeding & $\begin{array}{c}25 \\
(83.33)\end{array}$ & $\begin{array}{c}5 \\
(16.66)\end{array}$ & 0 & 84.90 & $\begin{array}{c}19 \\
(63.33)\end{array}$ & $\begin{array}{c}9 \\
(30.00)\end{array}$ & $\begin{array}{c}2 \\
(6.66)\end{array}$ & 64.11 \\
\hline 2 & Feeding & $\begin{array}{c}22 \\
(73.33)\end{array}$ & $4(13.33)$ & $\begin{array}{c}4 \\
(13.33)\end{array}$ & 83.33 & $\begin{array}{c}17 \\
(56.66)\end{array}$ & $\begin{array}{c}3 \\
(10.00)\end{array}$ & $\begin{array}{c}10 \\
(33.33)\end{array}$ & 59.39 \\
\hline 3 & Health Care & $\begin{array}{c}23 \\
(76.66)\end{array}$ & $\begin{array}{c}7 \\
(23.33)\end{array}$ & 0 & 78.66 & $\begin{array}{c}11 \\
(36.66)\end{array}$ & $\begin{array}{c}11 \\
(36.66)\end{array}$ & $\begin{array}{c}8 \\
(26.66)\end{array}$ & 50.00 \\
\hline 4 & $\begin{array}{l}\text { Milk } \\
\text { Production }\end{array}$ & $\begin{array}{c}22 \\
(73.33)\end{array}$ & $\begin{array}{c}6 \\
(20.00)\end{array}$ & $\begin{array}{c}2 \\
(6.66)\end{array}$ & 82.85 & $\begin{array}{c}13 \\
(43.33)\end{array}$ & $\begin{array}{c}8 \\
(26.66)\end{array}$ & $\begin{array}{c}9 \\
(30.00)\end{array}$ & 60.47 \\
\hline \multicolumn{2}{|c|}{ Over all } & $\begin{array}{c}24 \\
(80.00)\end{array}$ & $\begin{array}{c}6 \\
(20.00)\end{array}$ & - & 84.79 & $\begin{array}{c}13 \\
(43.33)\end{array}$ & $\begin{array}{c}10 \\
(33.33)\end{array}$ & $\begin{array}{c}7 \\
(23.33)\end{array}$ & 59.21 \\
\hline
\end{tabular}

Table.7 Distribution of the respondents by the adoption of improved practices of Livestock Development $n=60$

\begin{tabular}{|c|c|c|c|c|c|c|c|c|c|}
\hline \multirow[t]{3}{*}{ S. No } & \multirow[t]{3}{*}{ Components } & \multicolumn{8}{|c|}{ Extent of Adoption } \\
\hline & & \multicolumn{4}{|c|}{ Beneficiaries $n_{1}=30 \mathrm{f}(\%)$} & \multicolumn{4}{|c|}{ Non - Beneficiaries $n_{2}=30 \mathrm{f}(\%)$} \\
\hline & & High & Medium & Low & MPS & High & Medium & Low & MPS \\
\hline 1 & Breeding & $\begin{array}{c}25 \\
(83.33)\end{array}$ & $\begin{array}{c}5 \\
(16.66)\end{array}$ & 0 & 81.33 & $\begin{array}{c}9 \\
(30.00)\end{array}$ & $\begin{array}{c}19 \\
(63.33)\end{array}$ & $\begin{array}{c}2 \\
(6.66)\end{array}$ & 58.00 \\
\hline 2 & Feeding & $\begin{array}{c}17 \\
(56.66)\end{array}$ & $\begin{array}{c}13 \\
(43.33)\end{array}$ & 0 & 72.00 & $\begin{array}{c}4 \\
(13.33)\end{array}$ & $\begin{array}{c}24 \\
(80.00)\end{array}$ & $\begin{array}{c}2 \\
(6.66)\end{array}$ & 50.33 \\
\hline 3 & Health Care & $\begin{array}{c}24 \\
(80.00)\end{array}$ & $\begin{array}{c}6 \\
(20.00)\end{array}$ & 0 & 81.66 & $\begin{array}{c}12 \\
(40.00)\end{array}$ & $\begin{array}{c}14 \\
(46.66)\end{array}$ & $\begin{array}{c}4 \\
(13.33)\end{array}$ & 58.66 \\
\hline 4 & Milk Production & $\begin{array}{c}13 \\
(43.33)\end{array}$ & $\begin{array}{c}16 \\
(53.33)\end{array}$ & $\begin{array}{c}1 \\
(3.33)\end{array}$ & 71.94 & $\begin{array}{c}10 \\
(33.33)\end{array}$ & $\begin{array}{c}19 \\
(63.33)\end{array}$ & $\begin{array}{c}1 \\
(3.33)\end{array}$ & 59.66 \\
\hline & Over all & $\begin{array}{c}17 \\
(56.66)\end{array}$ & $\begin{array}{c}13 \\
(43.33)\end{array}$ & $\mathbf{0}$ & 75.80 & $\begin{array}{c}6 \\
(20.00)\end{array}$ & $\begin{array}{c}24 \\
(80.00)\end{array}$ & $\mathbf{0}$ & 55.40 \\
\hline
\end{tabular}


Table.8 Distribution of respondents by their opinion towards Livestock Development $n=30$

\begin{tabular}{|c|c|c|c|c|}
\hline $\begin{array}{l}\text { S. } \\
\text { No }\end{array}$ & Statements & $\begin{array}{c}\text { Agree } \\
\mathbf{f}(\%)\end{array}$ & $\begin{array}{c}\text { Undecided } \\
\mathbf{f}(\%)\end{array}$ & $\begin{array}{c}\text { Disagree } \\
\mathbf{f}(\%)\end{array}$ \\
\hline 1. & Male cross bred calf is of no use & $\begin{array}{c}4 \\
(13.33)\end{array}$ & $\begin{array}{c}4 \\
(13.33)\end{array}$ & $\begin{array}{c}22 \\
(73.33)\end{array}$ \\
\hline 2. & A.I is an unnatural method & $\begin{array}{c}5 \\
(16.66)\end{array}$ & $\begin{array}{c}20 \\
(66.66)\end{array}$ & $\begin{array}{c}5 \\
(16.66)\end{array}$ \\
\hline 3. & Insemination charges are high & $\begin{array}{c}6 \\
(20.00)\end{array}$ & $\begin{array}{c}6 \\
(20.00)\end{array}$ & $\begin{array}{c}18 \\
(60.00)\end{array}$ \\
\hline 4. & Livestock management is a skilled job & $\begin{array}{c}17 \\
(56.66)\end{array}$ & $\begin{array}{c}5 \\
(16.66)\end{array}$ & $\begin{array}{c}8 \\
(26.66)\end{array}$ \\
\hline 5. & Milk production is more in crossbred & $\begin{array}{c}27 \\
(90.00)\end{array}$ & $\begin{array}{c}2 \\
(6.66)\end{array}$ & $\begin{array}{c}1 \\
(3.33)\end{array}$ \\
\hline 6. & Not a profitable venture & $\begin{array}{c}4 \\
(13.33)\end{array}$ & $\begin{array}{c}10 \\
(33.33)\end{array}$ & $\begin{array}{c}16 \\
(53.33)\end{array}$ \\
\hline 7. & Feed requirement is more in crossbred cow & $\begin{array}{c}5 \\
(16.66)\end{array}$ & $\begin{array}{c}21 \\
(70.00)\end{array}$ & $\begin{array}{c}4 \\
(13.33)\end{array}$ \\
\hline 8. & Generate employment & $\begin{array}{c}2 \\
(6.66)\end{array}$ & $\begin{array}{c}26 \\
(86.66)\end{array}$ & $\begin{array}{c}2 \\
(6.66)\end{array}$ \\
\hline 9. & Labour intensive activities. & $\begin{array}{c}22 \\
(73.33)\end{array}$ & $\begin{array}{c}2 \\
(6.66)\end{array}$ & $\begin{array}{c}6 \\
(20.00)\end{array}$ \\
\hline 10. & A.I is a tedious process & $\begin{array}{c}4 \\
(13.33)\end{array}$ & $\begin{array}{c}12 \\
(40.00)\end{array}$ & $\begin{array}{c}14 \\
(46.66)\end{array}$ \\
\hline 11. & Education is important to rear a cattle & $\begin{array}{c}26 \\
(86.66)\end{array}$ & $\begin{array}{c}2 \\
(6.66)\end{array}$ & $\begin{array}{c}2 \\
(6.66)\end{array}$ \\
\hline 12. & Improves health and nutritional status & $\begin{array}{c}19 \\
(63.33)\end{array}$ & $\begin{array}{c}5 \\
(16.66)\end{array}$ & $\begin{array}{c}6 \\
(20.00)\end{array}$ \\
\hline 13. & Diet affects milk production & $\begin{array}{c}30 \\
(100)\end{array}$ & 0 & 0 \\
\hline 14. & $\begin{array}{l}\text { Cross bred cattle is more susceptible to } \\
\text { diseases as to deshibreds of cow }\end{array}$ & $\begin{array}{c}23 \\
(76.66)\end{array}$ & $\begin{array}{c}4 \\
(13.33)\end{array}$ & $\begin{array}{c}3 \\
(10.00)\end{array}$ \\
\hline 15. & $\begin{array}{l}\text { Timely vaccination and deworming protects } \\
\text { animal health }\end{array}$ & $\begin{array}{c}30 \\
(100)\end{array}$ & 0 & 0 \\
\hline 16. & Green fodder is best for cattles & $\begin{array}{c}21 \\
(70.00)\end{array}$ & $\begin{array}{c}5 \\
(16.66)\end{array}$ & $\begin{array}{c}4 \\
(13.33)\end{array}$ \\
\hline
\end{tabular}


Table.9 Constraints faced by the beneficiaries of Livestock Development Centre $n=30$

\begin{tabular}{|c|c|c|c|}
\hline S. No & Constraints & MPS & Rank \\
\hline 1 & \multicolumn{2}{|l|}{ Personal } & \multirow{9}{*}{ II } \\
\hline$\overline{\mathbf{A}}$ & Unavailability of land for green fodder production & 86.66 & \\
\hline B & Difficulty in maintaining records due to illiteracy & 86.66 & \\
\hline C & Lack of knowledge regarding dairy innovations & 86.66 & \\
\hline D & Irrigation facilities for growing green fodder & 83.33 & \\
\hline $\mathbf{E}$ & Reluctance in feeding balanced ration & 78.88 & \\
\hline $\mathbf{F}$ & Lack of knowledge for preparing balanced feed & 78.88 & \\
\hline $\mathbf{G}$ & High cost of cross bred cattle & 77.77 & \\
\hline & Over all & 82.69 & \\
\hline 2 & \multicolumn{2}{|l|}{ Technical } & \multirow{7}{*}{ III } \\
\hline A & $\begin{array}{l}\text { Insufficient knowledge of the important management } \\
\text { practices like deworming, castration, etc }\end{array}$ & 80.00 & \\
\hline B & Lack of knowledge about cattle diseases and control & 78.89 & \\
\hline C & Non availability of improved fodder seed & 76.66 & \\
\hline D & Lack of knowledge about scientific method of milking & 72.22 & \\
\hline $\mathbf{E}$ & Inadequate knowledge of breeding practices & 70.00 & \\
\hline & Over all & $\mathbf{7 5 . 5 6}$ & \\
\hline 3 & \multicolumn{2}{|l|}{ Economic } & \multirow{13}{*}{ I } \\
\hline A & High cost of veterinary medicines & 93.33 & \\
\hline $\bar{B}$ & $\begin{array}{l}\text { Lack of credit facilities for purchase cattle feed and } \\
\text { mineral mixture }\end{array}$ & 85.55 & \\
\hline C & High cost of concentration & 82.22 & \\
\hline D & Distance and location of A.I centres & 71.11 & \\
\hline & Over all & 83.05 & \\
\hline 4 & \multicolumn{2}{|l|}{ General } & \\
\hline A & Lack of educational programme on cattle rearing & 81.11 & \\
\hline B & $\begin{array}{l}\text { Lack of information about government programmes and } \\
\text { facilities provided for cattle keepers }\end{array}$ & 77.77 & \\
\hline C & Unavailability of bank facilities for loan & 71.11 & \\
\hline D & Non availability of improved fodder seed & 70.00 & \\
\hline $\mathbf{E}$ & Lack of retail fodder shop & 61.11 & \\
\hline $\mathbf{F}$ & $\begin{array}{l}\text { Lack of veterinary hospital and health centers in/around } \\
\text { the village }\end{array}$ & 54.44 & \\
\hline & Over all & 69.25 & IV \\
\hline
\end{tabular}

Akhter (2014) reported alike where knowledge level of farmer's in case of breeding of dairy animals regarding artificial insemination was found maximum $(91.1 \%)$ as compared to the breeding of cows $(86.7 \%)$ and buffalo (53.3\%). In case of feeding practices colostrums feeding (93.3\%), leguminous green fodder $(92.2 \%)$, use of mangers $(90.0 \%)$, chaffing of fodder $(87.8 \%)$, feeding of concentrates during lactation (77.8\%), and feeding of concentrates during pregnancy $(66.7 \%)$. The knowledge of the 
respondents regarding health and hygiene, approximately 50 percent of the respondents were aware about the deworming (62.2\%), control of external parasites $(50.0 \%)$ and proper time of vaccination $(47.8 \%)$. The study further indicates that knowledge of animal owners (Farmers) regarding animal husbandry practices was observed for clean milk production $(82.6 \%)$, followed by breeding of dairy animals $(78.9 \%)$, feeding of dairy animals $(78.0 \%)$ and Health and hygiene $(53.3 \%)$. The overall results revealed that the farmers were found more familiar to all animal husbandry practices (50 to $80 \%$ ).

Adoption of improved practices of Livestock Development service by the respondents

In adoption of the service, the recommended practices adopted by the respondents was studied and presented in Table 7. In the service, respondents were categorized in high, medium and low adoption categories along with MPS.

Data presented in Table 7 reveal the adoption of livestock development. In adoption of Livestock Development service beneficiaries had high extent of adoption with 75.80 MPS. Distribution of the respondents also reveals that 56.66 per cent beneficiaries were in high adoption category and 43.33 per cent beneficiaries were in medium adoption category.

In case of non-beneficiaries, MPS 55.40 in Livestock Development indicates medium extent of adoption also revealed by the data as 80.00 per cent of the non-beneficiaries were in medium adoption category and only 20.00 per cent of the non-beneficiaries were in high adoption category.

High extent of adoption was found among beneficiaries in all the components viz breeding, feeding, health care and milk production also indicated by MPS as 81.33 , $72.00,81.66$ and 71.94 , respectively.

Further, in depth review of Table 7 indicates that in breeding component 83.33 per cent beneficiaries were in high adoption category and 16.67 per cent beneficiaries were in medium adoption category with MPS 81.33. In feeding component 56.66 per cent and 43.33 per cent beneficiaries were in high and medium adoption category respectively, with MPS 72.00. In health care component, 80.00 per cent beneficiaries were in high adoption category and MPS 81.66. In milk production component, 53.33 per cent beneficiaries were in medium extent of adoption and 43.33 per cent beneficiaries were in high adoption category and MPS 71.94.

In case of non-beneficiaries, medium extent of adoption for livestock Development as depicted by MPS 58.00, 50.33, 58.66 and 59.66 in breeding, feeding, health care and milk production respectively. The table further shows that in breeding component 63.33 per cent non beneficiaries were in medium adoption category and 30.00 percent beneficiaries in high adoption category. In feeding component 80.00 per cent non beneficiaries were falling in medium adoption category while only 13.33 per cent and 6.66 per cent non beneficiaries were adopting it to high and poor extent respectively. Further 46.66 per cent non beneficiaries were in medium and 40.00 per cent non beneficiaries were in high adoption category in the healthcare component of Livestock Development. In milk production component, 63.33 per cent non beneficiaries were in medium adoption category followed by 33.33 per cent non beneficiaries in high adoption category.

Livestock Development is very beneficial service and beneficiaries continuously 
generating income by adopting its improved practices and very much involved with implementing agency in various activities like doing artificial insemination for their cattle and vaccinating them on particular time.

The above findings are supported by Vashishtha (2007) who reported that only 17 per cent respondents fed the cattle with balanced ration. A good number of respondents used to supply extra ration during pregnancy of cow (78\%) and after calving (92\%).

The Findings can be further supported by Kumar and Dangi (2012) who found that the adoption level of livestock interventions introduced under NAIP was reflected high among $79(75.96 \%)$ of beneficiaries, 22 (21.15\%) farmers belonged to moderate level of adoption. Furthermore, data clearly show that of total beneficiaries, $101 \quad(97.11 \%)$ expressed overwhelmingly satisfactory level of adoption.

\section{Opinion of beneficiaries towards livestock development services}

Opinion of the beneficiaries was studied towards the service promoted under TADP. The statement wise results are presented in Table 8.

Table 8 illustrate the opinion of beneficiaries towards Livestock Development, that 70.00 100 per cent of beneficiaries agree with the statements like milk production is more in crossbred, education is important to rear a cattle, diet affects milk production, timely vaccination and deworming protects animal health and green fodder is best for cattle. Reasons behind these findings may be due to the beneficiaries own experience in having more milk production with crossbred, help of education, timely vaccination, deworming and proper diet in milk production. Majority of the beneficiaries (76.66\%) agree that cross breed cattle is more susceptible to diseases as compared to deshi cows.

Further, insemination charges are high and livestock is not a profitable venture was disagreeing by 60.00 per cent and 53.33 cent of the beneficiaries respectively of the service. The table further reveals that in Livestock Development few beneficiaries were undecided towards A.I is an unnatural method $(66.66 \%)$, feed requirement is more in crossbred cow $(70.00 \%)$ and generate employment (86.66\%). With regard to A.I is a tedious process, 46.66 per cent and 40.00 per cent of the beneficiaries were undecided or in disagreement respectively. The findings indicate an agreement towards the service.

\section{Constraints faced by the beneficiaries of livestock development service}

The constraints were categorized as personal, technical, economic and general for the service and measured on three point continuum. The scores were converted to MPSs and accordingly ranks were given. The results of constraints are presented in Table 9. It was found that economic constraints (Rank I) were the most important constraint with MPS 83.05, next personal constraints which rank II with MPS 82.69, technical constraints were ranked III with 75.56 MPS followed by general constraints 69.25 MPS (rank IV).

The table further reveals the major personal constraints perceived by the Livestock Development beneficiaries, were unavailability of land for green fodder production (86.66 MPS), difficulty in maintaining records due to illiteracy (86.66 MPS), lack of knowledge regarding dairy innovations (86.66 MPS) and irrigation facilities for growing green fodder (83.33 MPS). The other major constraints experienced by the beneficiaries of the service 
were reluctance in feeding balanced ration (78.88 MPS) and lack of knowledge for preparing balanced feed (78.88 MPS) followed by high cost of cross bred cattle (77.77 MPS).

In case of technical constraints, it was found that all the technical constraints viz insufficient knowledge of the important management practices like deworming, castration, etc lack of knowledge about cattle diseases and control, non-availability of improved fodder seed, lack of knowledge about scientific method of milking and inadequate knowledge of breeding practices were faced by the beneficiaries of Livestock Development to great extent as the MPS ranges between $70.00-80.00$.

All the economic constraints were also faced to great extent by the beneficiaries high cost of veterinary medicines, lack of credit facilities for purchase of cattle feed and mineral mixture and high cost of concentrate followed by distance and location of A.I centres with MPS 93.33, 85.55, 82.22 and 71.11 respectively. Beneficiaries were having more problem of finance. More expenditure on animals and high cost lead to the most important constraints which must be considered by the agency worker and put forward more steps to drain out the constraints.

Major general constraints faced by the beneficiaries were found to be lack of educational programme on cattle rearing (81.11 MPS), lack of information about government programmes and facilities provided for cattle keepers (77.77 MPS) and unavailability of bank facilities for loan (71.11 MPS) followed by non-availability of improved fodder seed (70.00 MPS). Beneficiaries of the service faced constraints to some extent were lack of retail fodder shop (61.11 MPS) and lack of veterinary hospital and health centres in/around the village (54.44 MPS).

According to the study findings it can be concluded that, majority of the beneficiaries possessed good or average knowledge and non-beneficiaries possessed average or poor knowledge in various components of livestock development Service. In adoption of the service, majority of the beneficiaries were in high and medium adoption categories in various components of all the service while in case of non-beneficiaries all were in medium or low adoption categories in the service. Opinion towards the service reveals favourable opinion of beneficiaries towards the livestock development service. All the beneficiaries reported for personal, technical, economic and general constraints for each of the service to great extent.

Based on these findings it can be said that there is need to reduce the constraints as far as possible and increase the knowledge with adoption of various components of the service for sustainable development with support of favourable opinion.

\section{References}

Akhter, J., Asiwal, B. L. and Hussain, A. 2013. Knowledge and Adoption of Animal Husbandry Practices among the Farmers of Sikar District of Rajasthan. Indian Journal of Extension Education and Rural Development. 21: 196-199.

Chaudhary, M., Singh, P. and Sharma, K.C. 2013. Constraints Faced By Farm Women in Adoption of Improved Cattle Management Practices in Arid Rajasthan. Indian Journal of Extension Education and Rural Development. 21: 153-158.

Kumar, R and Dangi, K.L. 2012. Effectiveness of NAIP in adoption of livestock based IFS interventions 
among the tribal beneficiaries. Rajasthan journal of Extension Education. 20

Padhy, K.S. and P. Chandra. 1989. Tribal India satapathy, Ashish Publishing house, New Delhi - 110026

Paul, S., Punjabi, N.K. and Paul, N. 2003. Constraints Encountered by the Tribal in Rearing Crossbreed Cattle. Rural India. 66: 27-31.

Rathore, R. S. 2014. Adoption of improved management practices by buffalo owners Indian Journal of Extension
Education \& Rural Development. 22: 67-71

Sure, D. 2015. January - June 2014. Tribal development through five year plans in India - an overview. www.thedawnjournal.com retrieved on 13 July 2015

Vashishtha, M. 2007. Adoption of improved dairy cattle management practices by rural women in Udaipur district, Rajasthan. An Unpublished M.Sc. thesis, MPUAT, Udaipur (Raj.).

\section{How to cite this article:}

Sonali Sharma, Snehlata Maheshwari, Rajshree Upadhyay, Bhupendra Upadhyay and Dangi, B.L. 2018. Over All Assessment of Tribals of Udaipur under Livestock Development Service of Tribal Area Development Programme. Int.J.Curr.Microbiol.App.Sci. 7(09): 317-329. doi: https://doi.org/10.20546/ijcmas.2018.709.039 